\title{
Evaluation of a new commercial assay for the detection of Rotavirus in stool samples
}

\author{
Maria Cristina Arcangeletti, Silvia Preti, Maria del Pilar Esteban, Valeria Albonetti, Emanuel Merep Djouvoup, \\ Giuseppe Dettori, Carlo Chezzi \\ Sezione di Microbiologia, Dipartimento di Patologia e Medicina di Laboratorio, Università degli Studi di Parma
}

Key words: Rotavirus; Laboratory diagnosis; Electropherotype; Immunochromatografic assay

Valutazione di un nuovo saggio commerciale per l’identificazione di Rotavirus in campioni di feci

\section{SUMMARY}

Rotaviruses belonging to the Reoviridae family, are the most common causative agents of severe diarrhoea in infants and young children. Rotaviruses are easily spread by hand-to-mouth contact with stool from an infected person. Although most children with rotavirus diarrhoea recover on their own, in same cases they become very ill with severe vomiting, diarrhoea and life-threatening loss of fluids that requires hospitalization. In this context, the availability of effective laboratory diagnostic methods is important to assure a correct and rapid identification of these agents and to control viral spread.

In this study 58 stool samples were analyzed by traditional virological methods used in diagnostic routine, such as electron microscopy (ME), a commercial assay based on latex agglutination for rotavirus proteins and the analysis of the electropherotype of rotavirus RNA segments (used only for those samples with a negative agglutination test). An additional method, the immunochromatographic assay RIDA ${ }^{\circledR} \mathrm{QUICK}$ Rotavirus (R-Biopharm) for the research of virus-specific proteins, was applied retrospectively on the same samples stored at $-80^{\circ} \mathrm{C}$. Among the 58 stool samples included in this study, 46/58 (79.3\%) were identified as reovirus-like by ME; 4 I/58 (70.6\%) of them resulted positive for rotavirus by the latex agglutination test. The 5 samples negative by the latter method were tested for the electropherotype analysis, that demonstrated the positivity for rotavirus genome segments. The immunochromatographic assay RIDA ${ }^{\circledR} \mathrm{QUICK}$ Rotavirus identified as positive all the 46 samples (yet found as reovirus-like by EM), including those positive by the agglutination method, as well as those resulted positive with the electropherotype analysis.

Taken together, these data support the effectiveness of the immunochromatographic test RIDA ${ }^{\circledR} \mathrm{QUICK}$ Rotavirus, that is not only a cheap and simple-to-use method, allowing an easy interpretation of results, but also showed a sensitivity possibly comparable to the molecular technique.

\section{INTRODUZIONE}

Il genere Rotavirus, appartenente alla famiglia Reoviridae, comprende virus a RNA (genoma costituito da 11 segmenti di RNA bicatenario) con morfologia rotondeggiante e di taglia caratteristica $(75-80 \mathrm{~nm})$, senza pericapside lipidico e con proteine organizzate in strati concentrici che riflettono una simmetria icosaedrica. Sono classificati in 7 sierogruppi (AG) sulla base delle differenze della proteina VP6, il maggior antigene capsidico gruppo-specifico.

I rotavirus sono ampiamente distribuiti in tutti i vertebrati per i quali rappresentano un'importante causa di enterite; essi sono responsabili di circa un milione di decessi nella popolazione infantile del terzo mondo. Mostrano un elevato tropismo d'ospite moltiplicandosi difficilmente in ospiti non naturali.

Le gastroenteriti da rotavirus rappresentano la causa più frequente di gastroenterite grave nel bambino (2), con esito letale soprattutto nei Paesi in via di sviluppo, dove la malattia è considerata dall’Organizzazione Mondiale della Sanità una vera e propria emergenza sanitaria. Si tratta di un'infezione endemica, sebbene i rotavirus siano occasionalmente associati a vere e proprie epidemie. Nei paesi a clima temperato l'infezione ha una stagionalità caratteristica con un picco invernale, mentre nei paesi tropicali il virus è presente tutto l'anno. La trasmissione avviene per via fecale-orale, ma è possibile anche la trasmissione per via respiratoria 0 per contatto $(2,6)$.

La quasi totalità della popolazione è esposta all'infezione da rotavirus entro i primi anni di vita.

I sierogruppi responsabili di infezioni nell’uomo sono A, B e $\mathrm{C}$, tra i quali il gruppo A è il più diffuso a livello mondiale, il $\mathrm{B}$ è stato isolato solo in Asia; per quel che riguarda il gruppo C, anch'esso originariamente confinato ai paesi asiatici, la sua presenza è stata attualmente segnalata anche in Europa ed in particolare, in Italia (5). All'interno del gruppo A, sulla base di risultati di neutralizzazione nei riguardi delle protei- ne VP4 e VP7, sono stati individuati 14 sierotipi (G1-G14). Attualmente 5 sierotipi virali del gruppo A (G1, G2, G3, G4, G9) sono i ceppi circolanti con maggiore frequenza, rappresentando circa il 95\% dei ceppi nel mondo, sebbene con una variabilità geografica, di stagione e fluttuazioni annuali considerevoli $(3,4)$. L'eterogeneità, frutto di sierotipi emergenti, e la continua modificazione del quadro epidemiologico dei rotavirus, sottolineano il bisogno di una continua sorveglianza, da attuare anche attraverso mezzi diagnostici di laboratorio rapidi ed efficaci.

Questo studio si è proposto di effettuare la valutazione di un saggio immunocromatografico commerciale, RIDA ${ }^{\circledR}$ QUICK Rotavirus della ditta R-Biopharm, per la ricerca di proteine specifiche di rotavirus umano in campioni di feci di soggetti in età pediatrica con gastroenterite, a confronto con le tecniche utilizzate quali mezzi diagnostici di routine presso l'U.O. di Virologia dell'Azienda Ospedaliero-Universitaria di Parma, rappresentate, in particolare, dalla microscopia elettronica e dal saggio rapido di agglutinazione al latice (7).

\section{MATERIALI E METODI}

Lo studio in oggetto è stato condotto su 58 campioni fecali, nell'ambito di quelli prelevati nel corso degli anni 2005-2009 da soggetti in età pediatrica con gastroenterite ricoverati presso reparti dell'Azienda Ospedaliero-Universitaria di Parma o ambulatoriali. Essi erano stati sottoposti ad indagini virologiche utilizzate in routine diagnostica, quali la ricerca dell'agente virale mediante microscopia elettronica (ME) e un saggio commerciale di agglutinazione al latice per la ricerca di proteine specifiche di rotavirus umani (applicato a campioni positivi per reovirus-simili in ME). In caso di agglutinazione negativa, era stato utilizzato un metodo molecolare che consente l'analisi del profilo elettroforetico dei segmenti genomici di rotavirus (elettroferotipo). Un terzo metodo rapido, applicato retrospettivamente agli stessi campioni conservati a $-80^{\circ} \mathrm{C}$, è rappresentato dal saggio immunocromatogra-

\section{Corresponding author: Maria Cristina Arcangeletti}

Microbiology Section, Department of Pathology and Laboratory Medicine - University of Parma

43100 Parma, Italy - Viale A. Gramsci I4 - Tel. +39 052I 988885 - Fax. +39 0521993620

E-mail: mariacristina.arcangeletti@unipr.it 
fico RIDA ${ }^{\circledR}$ QUICK Rotavirus della ditta R-Biopharm, per la ricerca di dell'antigene capsidico VP6 di rotavirus di gruppo A.

\section{Saggio Immunocromatografico}

RIDA ${ }^{\circledR}$ QUICK Rotavirus della ditta R-Biopharm è un saggio immunocromatografico monofase a flusso laterale per la rilevazione qualitativa di antigeni di rotavirus umano in campioni di feci. In questo saggio, anticorpi monoclonali specifici anti-rotavirus umano sono accoppiati a particelle di latice rosse; altri anticorpi specifici sono saldamente fissati alla membrana; su un'altra riga della stessa membrana sono fissati anticorpi antitopo legati a particelle di latice blu (controllo di reazione). Un’aliquota di sospensione fecale opportunamente trattata e chiarificata viene introdotta nella "finestra" di inserimento del campione, presente nell'apparato. Se il campione è positivo per rotavirus, antigeni specifici

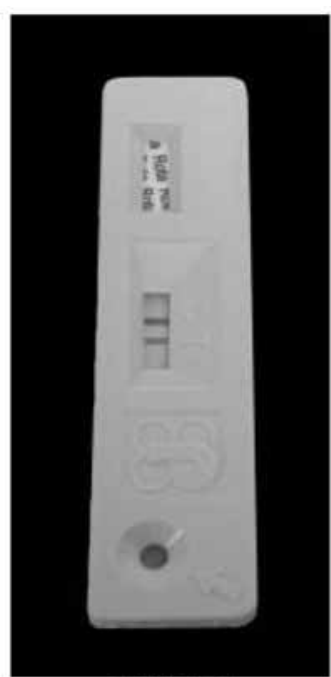

POSITIVO

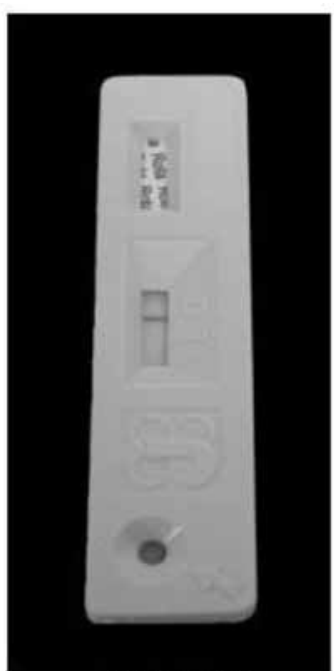

NEGATIVO

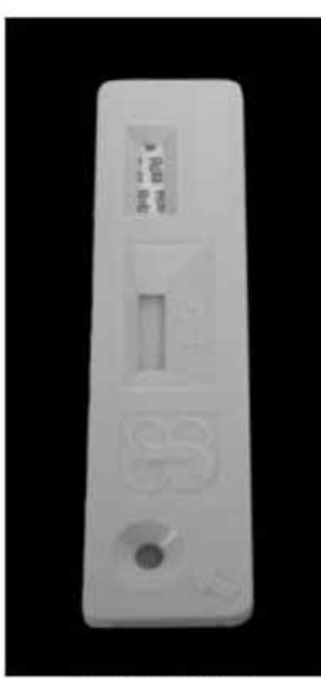

NON VALIDO
Figura I. Interpretazione dei risultati ottenuti mediante il saggio immunocromatografico RIDA ${ }^{\circledR}$ QUICK Rotavirus della ditta R-Biopharm. di tale agente si legheranno agli anticorpi coniugati alle particelle colorate di latice; il complesso migrerà attraverso la membrana, per poi legarsi alla banda specifica (a cui sono adesi anticorpi anti-rotavirus). In caso di positività, si svilupperà una banda di colore rosso (Figura I). Una ulteriore banda di colore blu segnalerà il corretto espletamento della procedura.

\section{RISULTATI}

Nell'ambito dei 58 campioni inclusi nello studio, 46 erano risultati positivi per reovirus-simili in ME (Figura II); 41/46 erano stati identificati come rotavirus mediante saggio di agglutinazione al latice mentre 5/46, negativi a quest'ultimo test, erano stati identificati come rotavirus mediante elettroferotipo. Il saggio immunocromatografico RIDA ${ }^{\circledR}$ QUICK Rotavirus è stato in grado di identificare come rotavirus tutti i 46 campioni positivi per reovirussimili in ME (Figura III). Per quel che riguarda i 12/58 campioni fecali negativi alla ME, di cui 6 positivi per altri agenti virali e 6 negativi per virus, il gruppo dei positivi per altri agenti virali si è confermato negativo per rotavirus con il saggio immunocromatografico. D’altra parte, nell'ambito dei 6 campioni negativi per agenti virali, 1/6

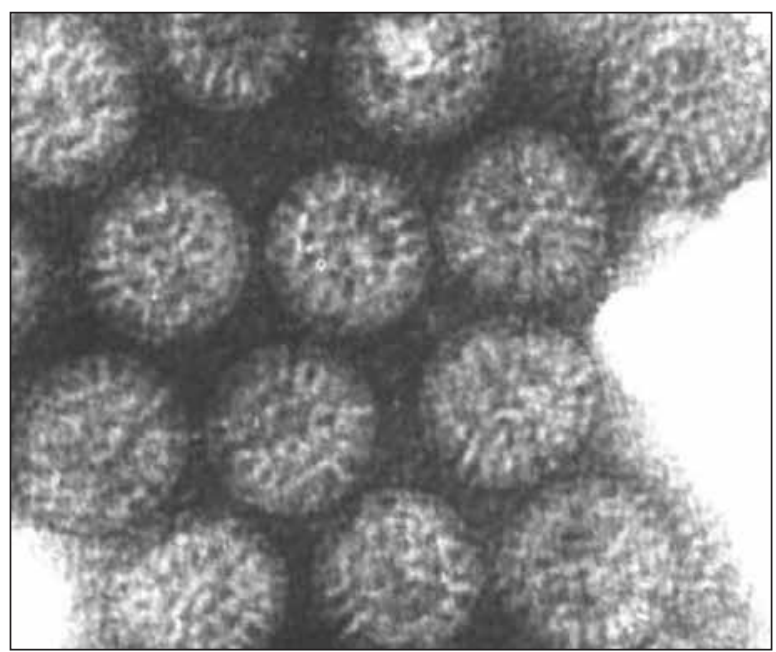

Figura II. Virioni di rotavirus al microscopio elettronico a trasmissione previa colorazione negativa.
(Figura III; indicato con un asterisco in Tabella 1) è risultato positivo per rotavirus con il suddetto metodo; esso è stato in seguito saggiato anche mediante agglutinazione al latice (indicato con un asterisco in Tabella 2), rivelandosi positivo. L'analisi statistica mediante tabella 2 X2 della performance del saggio immunocromatografico (Tabella 1) in riferimento alla ME, evidenzia una sensibilità analitica del $100 \%$, una specificità analitica del $91.7 \%$ ed una concordanza/accuratezza pari al 98,3\%. Applicando lo stesso approccio statistico, la performance del metodo di agglutinazione al latice (Tabella 2) in riferimento alla ME mostra una sensibilità analitica del 89.1\%, una specificità del $91.7 \%$ ed una concordanza/accuratezza pari al 89.6\%.

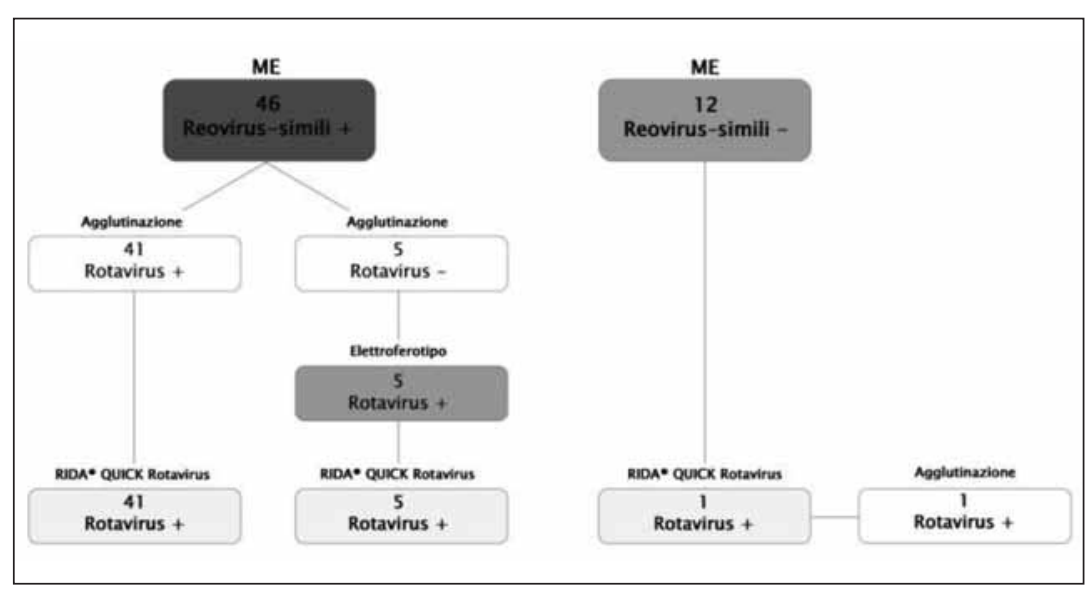

Figura III. Grafico riassuntivo delle indagini eseguite sui 58 campioni fecali selezionati per lo studio, relativamente alla diagnosi di infezione da rotavirus con le tecniche diagnostiche di routine e con il saggio RIDA ${ }^{\circledR}$ QUICK Rotavirus. 


\section{DISCUSSIONE}

Il saggio immunocromatografico RIDA ${ }^{\circledR}$ QUICK Rotavirus della ditta R-Biopharm è dotato di elevata sensibilità, è di facile impiego e di agevole lettura. La sua performance è apparsa migliore rispetto a quella del saggio di agglutinazione rapida in uso, non solo per una maggiore sensibilità, ma anche per una più immediata interpretazione dei risultati, facilmente discernibili come bande colorate rispetto ad una possibile, erronea interpretazione dell'agglutinazione, in particolare nei casi di scarsa concentrazione virale. Inoltre, il saggio RIDA ${ }^{\circledR}$ QUICK Rotavirus si è dimostrato in grado di riconoscere campioni risultati positivi solo attraverso il saggio molecolare (determinazione dell'elettroferotipo). D'altra parte, un limite del saggio, peraltro comune alla maggior parte dei test rapidi commerciali, è il fatto di non riconoscere rotavirus non appartenenti al gruppo A (1).

Tali caratteristiche, unitamente alla semplicità di esecuzione del saggio, lo rendono facilmente realizzabile anche da personale non esperto e/o operante in strutture prive di attrezzature tecnologicamente avanzate, per studi epidemiologici e per una diagnostica di screening di prima istanza, da confermare, eventualmente, mediante metodi molecolari (1).

Tabella I. Saggio immunocromatografico RIDA ${ }^{\circledR}$ QUICK Rotavirus (*Campione di feci negativo in ME e risultato positivo mediante RIDA ${ }^{\circledR}$ QUICK Rotavirus).

Sensibilità analitica: 100\%; Specificità analitica: 91.7\%; Concordanza/accuratezza: $98.3 \%$.

\begin{tabular}{|l|c|c|c|}
\hline $\begin{array}{l}\text { RIDA® QUICK } \\
\text { Rotavirus }\end{array}$ & \multicolumn{3}{|c|}{$\begin{array}{c}\text { ME } \\
\text { (reovirus-simili) }\end{array}$} \\
\hline $\mathbf{P}$ & $\mathbf{P}$ & $\mathbf{N}$ & TOT \\
\hline $\mathbf{N}$ & 46 & 1 * & 47 \\
\hline TOT & 0 & 11 & 11 \\
\hline & 46 & 12 & 58 \\
\hline
\end{tabular}

Tabella 2. Saggio di agglutinazione al latice per Rotavirus (*Campione di feci negativo in ME e risultato positivo mediante saggio di agglutinazione al latice).

Sensibilità analitica: 89.1\%; Specificità analitica: $91.7 \%$; Concordanza/accuratezza: $89.6 \%$.

\begin{tabular}{|l|c|c|c|}
\hline $\begin{array}{l}\text { Agglutinazione al } \\
\text { latice }\end{array}$ & \multicolumn{3}{|c|}{$\begin{array}{c}\text { ME } \\
\text { (reovirus-simili) }\end{array}$} \\
\hline & P & N & TOT \\
\hline P & 41 & 1 * & 42 \\
\hline N & 5 & 11 & 16 \\
\hline TOT & 46 & 12 & 58 \\
\hline & \multicolumn{2}{|l|}{$\begin{array}{l}\text { Interpretazione dei risultati } \\
\text { P= positivo; N= negativo }\end{array}$} \\
\hline
\end{tabular}

\section{BIBLIOGRAFIA}

1. Anderson EJ, Weber SG. Rotavirus infection in adults. The Lancet Infectious Diseases 2004; 4: 91-9.

2. Chandran A, Heinzen RR, Mathuram Santosham, Siberry GK. Nosocomial rotavirus infections: a systematic review. The Journal of Pediatrics 2006; 149: 441-7.

3. Hatab EA, Hussein HA, El-Sabagh IM, Saber MS. Isolation and antigenic and molecular characterization of G10 of group A rotavirus in camel. International Journal of Virology 2009; 5: 18-27.

4. Kapikian AZ, Chanok RM. Rotaviruses Field's Virology, Microbiological observation made on spontanous cases of acute neonatal calf diarrhea. Canadian Journal of Comparative Medicine 1996; 40: 228-40.

5. Medici MC, Abelli LA, Martinelli M, Martella V, Dettori G, Chezzi C. Molecular characterization of group $C$ rotaviruses detected in children in Italy. Journal of Clinical Virology 2009; 44 (1): 62-5.

6. Narkeviciute I, Tamusauskaite I. Peculiarities of norovirus and rotavirus infections in hospitalised young children. Journal of Pediatric Gastroenterology and Nutrition 2008; 46: 289-92.

7. Wilhelmi I, Colomina J, Martín-Rodrigo D, Roman E, Sánchez-Fauquier A. New immunochromatographic method for rapid detection of rotaviruses in stool samples compared with standard enzyme immunoassay and latex agglutination techniques. European Journal of Clinical and Microbiology Infectious Diseases 2001; 20: 741-3. 